\title{
Vielversprechende Langzeitergebnisse zum Einsatz von Anastrozol zur Prävention von Brustkrebs bei postmenopausalen Hochrisikopatientinnen
}

\author{
Josephin Trabitzsch ${ }^{1} \cdot$ Hendrik Schenke $^{1}$ \\ Online publiziert: 23. Juni 2020 \\ (c) Der/die Autor(en) 2020
}

Hintergrund und Ziel der Arbeit Nachdem für selektive Östrogenrezeptormodulatoren (SERM), wie Tamoxifen, bereits eine signifikante Langzeitwirkung in der Brustkrebsprävention bei Frauen mit einem hohen Risiko für Brustkrebserkrankungen nachgewiesen werden konnte [1], wurde in der IBIS-II-Studie die präventive Wirkung des Aromataseinhibitors (AI) Anastrozol bei postmenopausalen Risikopatientinnen untersucht. Erste Ergebnisse aus dem Jahr 2014 hatten eine Abnahme der Gesamtinzidenz von Brustkrebserkrankungen um 53\% in den ersten fünf Jahren nach Therapiebeginn gezeigt [2]. Die aktuelle Publikation ist ein Update der Studie mit längerem Follow-up.

Patientinnen und Methoden Bei der IBIS-II-Studie handelt es sich um eine internationale, randomisierte, doppelt verblindete und placebokontrollierte Interventionsstudie. Es wurden 3851 postmenopausale Frauen im Alter von 40 bis 70 Jahren mit einem mindestens 1,5-fach erhöhten Brustkrebsrisiko eingeschlossen. Die Interventionsgruppe erhielt fünf Jahre lang täglich $1 \mathrm{mg}$ Anastrozol. Primärer Endpunkt war die histopathologisch bestätigte Brustkrebsdiagnose im Verlauf. Als sekundäre Endpunkte galten sonstige Krebserkrankungen sowie ernste unerwünschte Reaktionen (Knochenfrakturen, Myokardinfarkte, tiefe Venenthrombosen, Lungenembolie, transiente ischämische Attacken, Schlaganfälle) und Tod. Nach Therapieabschluss wurden jährlich die Daten zu den Endpunkten erhoben. Weniger

Originalpublikation Cuzick J, Sestak I, Forbes JF, Dowsett M, Cawthorn S, Mansel RE et al (2020) Use of anastrozole for breast cancer prevention (IBIS-II). Long-term results of a randomised controlled trial. Lancet 395(10218):117-122. https://doi.org/10. 1016/S0140-6736(19)32955-1

Cand. med. Josephin Trabitzsch

j.trabitzsch@posteo.de

1 Klinik für Strahlentherapie, Universitätsklinikum Schleswig-Holstein, Campus Kiel, Feldstr. 21, 24105 Kiel, Deutschland ernste Nebenwirkungen wurden nicht weiter berücksichtigt. Zur Analyse der Endpunkte wurden Hazard Ratios basierend auf dem Cox-Regressionsmodell mit korrespondierenden $95 \%$-Konfidenzintervallen sowie eine Überlebenszeitanalyse nach Kaplan und Meier durchgeführt.

Ergebnisse Nach einem medianen Follow-up von 131 Monaten konnte eine Reduktion des Auftretens von Brustkrebs um $49 \%$ (HR 0,51, 95\%-KI 0,39-0,66, $p<0,0001$ ) nachgewiesen werden. Die Reduktion betrug in den ersten fünf Jahren des Follow-ups $61 \%$ (HR 0,39, $95 \%$-KI 0,27-0,58, $p<0,0001$ ), in den darauffolgenden Jahren $37 \%$ (HR 0,64, KI $0,45-0,91, p=0,014)$ und von Östrogenrezeptor-positiven invasiven Karzinomen $54 \%$. Bezüglich ernster unerwünschter Ereignisse wurden in den beiden Kohorten keine Unterschiede festgestellt. Insgesamt starben während des Follow-ups 69 Patientinnen der Interventionsgruppe und 70 Patientinnen der Placebogruppe. Da hiervon in der Interventionsgruppe nur 3 bzw. in der Anastrozolgruppe nur 2 Patientinnen an Brustkrebs verstarben, konnte bezüglich einer Letalitätssenkung durch den AI kein signifikanter Unterschied nachgewiesen werden.

Schlussfolgerung der Autoren Der in der ersten Publikation der Studie festgestellte Rückgang an Brustkrebserkrankungen durch Anastrozol hält auch nach mehr als 5 Jahren an. Die präventive Anastrozoleinnahme senkt also auch langfristig bei Frauen mit einem hohen Brustkrebsrisiko dieses Risiko nachhaltig, wobei die Nebenwirkungen geringer als diejenigen von Tamoxifen sind.

\section{Kommentar}

Brustkrebs ist die weltweit häufigste Krebserkrankung bei Frauen mit mehr als 2Mio. Neuerkrankungen im Jahr, z.B. 2018 [3]. Während die medikamentöse Prävention bei Hochrisikopatientinnen in den USA und in Großbri- 
tannien bereits Einzug in offizielle Therapieempfehlungen gefunden hat $[4,5]$, spielt sie in Deutschland bislang noch keine Rolle. Dabei wurde ein protektiver Effekt sowohl von Tamoxifen als auch von Aromataseinhibitoren längst beschrieben [6]. In einer Studie mit dem AI Exemestan wurden über einen Zeitraum von 5 Jahren ähnliche Ergebnisse wie hier mit Anastrozol erreicht [7].

Die Langzeitwirkung des Carry-over-Effekts einer präventiven Brustkrebstherapie wurde bislang lediglich für den selektiven Östrogenrezeptormodulator Tamoxifen untersucht [1]. Mit Tamoxifen wurde die Gesamtinzidenz von Brustkrebserkrankungen über 20 Jahre um gleichbleibend etwa $29 \%$ gesenkt. Die jetzt in der IBIS-II-Studie durch Anastrozol bewirkte Reduktion des Auftretens um $49 \%$ übertrifft somit die Ergebnisse mit Tamoxifen deutlich. Die NNT von Anastrozol in den ersten 12 Jahren nach Therapieabschluss schätzen die Autoren auf 29, während die von Tamoxifen bei 58 lag.

Während im Rahmen der Tamoxifenprophylaxe eine erhöhte Zahl an Karzinomen des Endometriums und auch thromboembolischen Ereignissen registriert wurde [1], konnten bei der Behandlung mit Anastrozol keine ernsten unerwünschten Reaktionen beobachtet werden. Dennoch darf man die während der Therapie signifikant erhöhten Risiken an muskuloskeletalen und vasomotorischen Beschwerden sowie an denen des ,trockenen Auges“ und des arteriellen Hypertonus [2] bei der Abwägung des NutzenRisiko-Verhältnisses nicht außer Acht lassen.

Die potenzielle Toxizität wiegt besonders schwer, da weiterhin keine Daten zur Mortalitätssenkung durch den Aromataseinhibitor vorliegen. Somit konnte auch die bereits 2014 geäußerte kritische Vermutung nicht entkräftet werden, es würde im Rahmen der Studie zu einer Überdiagnose und Überbehandlung von insbesondere hormonsensitiven, per Mammographie diagnostizierbaren Krebserkrankungen mit guter Prognose kommen [8].

\section{Fazit}

- Der Verdienst der Studie ist es, erstmals Langzeitergebnisse zum präventiven Einsatz von AI bei postmenopausalen Frauen der Brustkrebshochrisikogruppe zu liefern. Der Rückgang der Brustkrebsinzidenz ist auch $>5$ Jahre nach Absetzen des Medikaments noch immer signifikant und bedeutsam.

- Aufgrund der besseren Wirksamkeit und wegen des günstigeren Nebenwirkungsprofils scheint Anastrozol bei der Anwendung der pharmakologischen Prophylaxe von Brustkrebs bei postmenopausalen Hochrisikopatientinnen dem Tamoxifen überlegen zu sein.
- Für eine uneingeschränkte Empfehlung sind zunächst noch Langzeitdaten zur Letalität abzuwarten.

\section{Josephin Trabitzsch und Hendrik Schenke, Kiel}

Funding Open Access funding provided by Projekt DEAL.

Interessenkonflikt J. Trabitzsch und H. Schenke geben an, dass kein Interessenkonflikt besteht.

Open Access Dieser Artikel wird unter der Creative Commons Namensnennung 4.0 International Lizenz veröffentlicht, welche die Nutzung, Vervielfältigung, Bearbeitung, Verbreitung und Wiedergabe in jeglichem Medium und Format erlaubt, sofern Sie den/die ursprünglichen Autor(en) und die Quelle ordnungsgemäß nennen, einen Link zur Creative Commons Lizenz beifügen und angeben, ob Änderungen vorgenommen wurden.

Die in diesem Artikel enthaltenen Bilder und sonstiges Drittmaterial unterliegen ebenfalls der genannten Creative Commons Lizenz, sofern sich aus der Abbildungslegende nichts anderes ergibt. Sofern das betreffende Material nicht unter der genannten Creative Commons Lizenz steht und die betreffende Handlung nicht nach gesetzlichen Vorschriften erlaubt ist, ist für die oben aufgeführten Weiterverwendungen des Materials die Einwilligung des jeweiligen Rechteinhabers einzuholen.

Weitere Details zur Lizenz entnehmen Sie bitte der Lizenzinformation auf http://creativecommons.org/licenses/by/4.0/deed.de.

\section{Literatur}

1. Cuzick J, Sestak I, Cawthorn S et al (2015) Tamoxifen for prevention of breast cancer. Extended long-term follow-up of the IBIS-I breast cancer prevention trial. Lancet Oncol 16:67-75. https://doi. org/10.1016/S1470-2045(14)71171-4

2. Cuzick J, Sestak I, Forbes JF et al (2014) Anastrozole for prevention of breast cancer in high-risk postmenopausal women (IBIS-II). An international, double-blind, randomised placebocontrolled trial. Lancet 383:1041-1048. https://doi.org/10.1016/ S0140-6736(13)62292-8

3. Bray F, Ferlay J, Soerjomataram I et al (2018) Global cancer statistics 2018. GLOBOCAN estimates of incidence and mortality worldwide for 36 cancers in 185 countries. CA Cancer J Clin 68:394-424. https://doi.org/10.3322/caac.21492

4. NICE (2013) Familial breast cancer. Classification, care and managing breast cancer and related risks in people with a family history of breast cancer

5. Owens DK, Davidson KW, Krist AH et al (2019) Medication use to reduce risk of breast cancer. US preventive services task force recommendation statement. JAMA 322:857-867. https://doi.org/ 10.1001/jama.2019.11885

6. Cuzick J, Sestak I, Baum M et al (2010) Effect of anastrozole and tamoxifen as adjuvant treatment for early-stage breast cancer. 10-year analysis of the ATAC trial. Lancet Oncol 11:1135-1141. https://doi.org/10.1016/S1470-2045(10)70257-6

7. Goss PE, Ingle JN, Alés-Martínez JE et al (2011) Exemestane for breast-cancer prevention in postmenopausal women. N Engl J Med 364:2381-2391. https://doi.org/10.1056/NEJMoa1103507

8. Cameron DA (2014) Breast cancer chemoprevention. Little progress in practice? Lancet 383:1018-1020. https://doi.org/10.1016/ S0140-6736(13)62555-6 